\title{
UNIFORM SPACES AND WEAK SLICE SPACES
}

\author{
STEPHEN M. BUCKLEY AND DAVID A. HERRON
}

\begin{abstract}
We characterize uniform spaces in terms of a slice condition. We also establish the Gehring-Osgood-Väisälä theorem for uniformity in the metric space context.
\end{abstract}

\section{INTRODUCTION}

Uniformity in the metric space setting was introduced in [BHK01]; this generalizes the uniform domains in $\mathbf{R}^{n}$ whose importance in geometric analysis is well established as documented in [Geh87] and [Väi88]. Uniform domains in Euclidean space were first studied by John [Joh61] and Martio and Sarvas [MS79]. More recently, uniform subdomains of the Heisenberg groups, as well as more general Carnot groups, have become a focus of study; see [CT95], [CGN00], [Gre01]. Every bounded Lipschitz domain in $\mathbf{R}^{n}$ is uniform, but generic uniform domains may have fractal boundary.

The following characterization of uniform domains is a consequence of our main result, Theorem 4.2, which is our metric space version.

Theorem. An Euclidean domain $D \subsetneq \mathbf{R}^{n}, n \geq 2$, is uniform if and only if it is quasiconvex, LLC with respect to paths, and a weak slice domain. This equivalence is quantitative: the associated parameters depend only on each other, but not on $n$.

By definition, uniform domains are quasiconvex, and it is not difficult to see that they are LLC with respect to paths and satisfy a weak slice condition, so the significance of the above result is that these three conditions are sufficient for uniformity. One notable upshot of the above is the following.

Corollary. Suppose $D=G \backslash E$, where $G \subsetneq \mathbf{R}^{n}$ is a uniform domain and $E \subset G$ is closed and removable for the Sobolev space $W^{1, n}$. Then $D$ is uniform if and only if it is a weak slice domain.

This allows construction of nice domains that fail to satisfy a weak slice condition; for instance, simply remove a countable set from a uniform domain in such a way that the double cone condition is destroyed. For more on $W^{1, p}$ removability, see [Kos99] and the references cited therein.

This document is organized as follows: Section 2 contains preliminary information including basic definitions, notation, and terminology descriptions. There we

Received by the editors January 9, 2007.

2000 Mathematics Subject Classification. Primary 30C65; Secondary 51F99.

Key words and phrases. Uniform spaces, quasihyperbolic metric, slice conditions.

The first author was supported in part by Enterprise Ireland and Science Foundation Ireland. Both authors were supported by the Charles Phelps Taft Memorial Fund. 
also include Propositions 2.1 and 2.2 which may be of separate interest. In Section 3, we state our definition of uniform spaces, discuss some examples, and then establish Theorem 3.1 which is our metric space version of the Gehring-Osgood-Väisälä characterization for uniformity. In Section 4, we define the weak slice condition and prove our main result, Theorem 4.2, as well as the Theorem and Corollary mentioned above.

\section{Preliminaries}

2.A. General Information. Our notation is relatively standard. We write $C=$ $C(a, \ldots)$ to indicate a constant $C$ which depends only on the parameters $a, \ldots$ Typically $a, b, c, C, K, \ldots$ will be constants that depend on various parameters, and we try to make this as clear as possible, often giving explicit values. However, at times $C$ may denote some constant whose value depends only on the data present and may differ even in the same line of inequalities. For real numbers we employ the notation

$$
a \wedge b:=\min \{a, b\} \quad \text { and } \quad a \vee b:=\max \{a, b\} .
$$

Except where explicitly stated otherwise, $(X, d)$ is a metric space with no presumed special properties. We write $|x-y|:=d(x, y)$ for the distance between $x, y$. The metric completion of $(X, d)$ is $(\bar{X}, d)$ and $\partial X=\bar{X} \backslash X$ is the metric boundary of $X$. We let $B(x ; r):=\{y:|x-y|<r\}$ (and $S(x ; r):=\{y:|x-y|=r\}$ ) denote the open ball (and sphere) with center $x$ and radius $r$; also, $\lambda B(x ; r):=B(x ; \lambda r)$ for $\lambda>0$. We put

$$
d(x):=\operatorname{dist}(x, \partial X) \quad \text { and } \quad B(x):=B(x ; d(x)) .
$$

Given $\lambda \in(0,1 / 2]$, we call $\lambda B(x)=B(x ; \lambda d(x))$ a Whitney ball at $x$ with parameter $\lambda$.

In the above, we are tacitly assuming that $(X, d)$ is locally complete which simply means that for all $x \in X, d(x)>0$; equivalently, $\partial X$ is closed in $\bar{X}$. All locally compact spaces are locally complete. Subdomains of infinite dimensional Banach spaces are locally complete but not necessarily locally compact.

Throughout this paper, $d, l$, and $k$ will denote three metrics related to each other in a special way. The space $(X, d)$ will often be a rectifiably connected, incomplete, locally complete metric space; we call such a space minimally nice. The metric $l$, defined below, is the length metric (also called the inner or intrinsic metric) associated with $d$, and $k$ (discussed in $\S 2 . \mathrm{C}$ ) is the associated quasihyperbolic metric. Minimally nice spaces include all Euclidean domains $D \subsetneq \mathbf{R}^{n}$ as well as domains in Banach spaces.

An arc is the homeomorphic image of an interval, and it is open or closed if the interval is open or closed, respectively. When $x, y$ are points on an arc $\alpha$ we write $\alpha(x, y)$ and $\alpha[x, y]$ to denote the open and closed subarcs of $\alpha$ between $x$ and $y$, respectively. If $\gamma$ is merely a path, then $\gamma[x, y]$ refers to a fixed but arbitrary subpath of $\gamma$ with endpoints $x, y$.

The length of a (continuous) path $\gamma:[0,1] \rightarrow X$ is defined, in the usual way by

$$
\ell(\gamma):=\sup \sum_{i=1}^{n}\left|\gamma\left(t_{i}\right)-\gamma\left(t_{i-1}\right)\right| \quad \text { where } 0=t_{0}<t_{1}<\cdots<t_{n}=1
$$


We call $\gamma$ rectifiable when $\ell(\gamma)<\infty$. We let $\Gamma(x, y)=\Gamma(x, y ; X)$ denote the collection of all rectifiable paths joining $x$ to $y$ in $X$. Then $X$ is rectifiably connected precisely when $\Gamma(x, y) \neq \emptyset$ for all $x, y \in X$.

A geodesic in $X$ is the image $\varphi(I)$ of some isometric embedding $\varphi: I \rightarrow X$ where $I \subset \mathbf{R}$ is an interval; we use the adjectives segment, ray, or line (respectively) to indicate that $I$ is bounded, semi-infinite, or all of $\mathbf{R}$. When $\varphi$ is bilipschitz, we call $\varphi(I)$ a quasigeodesic.

Every rectifiably connected metric space $(X, d)$ admits a natural (or intrinsic) metric, its so-called length distance given by

$$
l(x, y):=\inf \{\ell(\gamma): \gamma \text { a rectifiable curve joining } x, y \text { in } \Omega\} .
$$

A metric space $(X, d)$ is a length space provided $d(x, y)=l(x, y)$ for all points $x, y \in X$; it is also common to call such a $d$ an intrinsic distance function. Notice that a length geodesic is a shortest curve joining $x$ and $y$, but it need not be a distance geodesic.

We require the following result which may be of independent interest; it is a handy replacement for not having quasigeodesics.

2.1. Proposition. Suppose $(X, l)$ is a length space. Let $c>1$. Then for all $x, y \in X$, there exists a path $\gamma \in \Gamma(x, y)$ satisfying $\ell(\gamma) \leq \sqrt{c} l(x, y)$ and with the property that

$$
\forall z \in \gamma, \forall w \in\{x, y\}: \quad \ell(\gamma[w, z]) \leq \operatorname{cl}(w, z) .
$$

Proof. Let $x, y \in X$. We construct $\gamma$ as a limit of paths $\left[t_{n}, s_{n}\right] \stackrel{\sigma_{n}}{\rightarrow} X$ where $\left[t_{n-1}, s_{n-1}\right] \subsetneq\left[t_{n}, s_{n}\right] \subsetneq[0,1], \sigma_{n}=\left.\sigma_{n+1}\right|_{\left[t_{n}, s_{n}\right]}, a_{n}=\sigma_{n}\left(t_{n}\right) \rightarrow x, b_{n}=\sigma_{n}\left(s_{n}\right) \rightarrow$ $y$, and the desired property holds for all points $z$ on each $\sigma_{n}$.

Put $L=l(x, y), b=\sqrt{c}$ and $R_{n}=L / b^{n}$. Select a path $[0,1] \stackrel{\gamma_{0}}{\rightarrow} X$ in $\Gamma(x, y)$ with $\gamma_{0}(0)=x, \gamma_{0}(1)=1$ and $\ell\left(\gamma_{0}\right) \leq b L$. Define

$t_{1}:=\sup \left\{t \in[0,1]: l\left(\gamma_{0}(t), x\right)=R_{1}\right\} \quad$ and $\quad s_{1}:=\inf \left\{t \in[0,1]: l\left(\gamma_{0}(t), y\right)=R_{1}\right\}$.

Then $\sigma_{1}:=\left.\gamma_{0}\right|_{\left[t_{1}, s_{1}\right]}$ is a subpath of $\gamma_{0}$ with endpoints $a_{1}:=\gamma_{0}\left(t_{1}\right), b_{1}:=\gamma_{0}\left(s_{1}\right)$ and $\sigma_{1}$ lies outside $B\left(x ; R_{1}\right) \cup B\left(y ; R_{1}\right)$. We claim that if $\gamma \in \Gamma(x, y)$ satisfies $\sigma_{1} \subset \gamma$ and $\ell(\gamma) \leq b L$, then

$$
\forall z \in \sigma_{1}, \forall w \in\{x, y\}: \quad \ell(\gamma[w, z]) \leq \operatorname{cl}(w, z) .
$$

Indeed, given such points $z, w$ on such a path $\gamma$, we have $l(w, z) \geq R_{1}$ and so

$$
\ell(\gamma[w, z]) \leq \ell(\gamma) \leq b L=b^{2} R_{1} \leq \operatorname{cl}(w, z) .
$$

In particular, the above holds for the path $\gamma_{0}$.

Now we modify $\gamma_{0}$ to obtain a path $[0,1] \stackrel{\gamma_{1}}{\rightarrow} X$ in $\Gamma(x, y)$ as follows: $\gamma_{1}$ is the concatenation of paths $\alpha_{1}, \sigma_{1}, \beta_{1}$ where $\alpha_{1}:\left[0, t_{1}\right] \rightarrow X, \beta_{1}:\left[s_{1}, 1\right] \rightarrow X$ are paths in $\Gamma\left(x, a_{1}\right), \Gamma\left(y, b_{1}\right)$ (respectively) which are chosen to have lengths at most $b R_{1}$, and we use appropriate subpaths of $\gamma_{0}$ if these are short enough. Thus $\ell\left(\gamma_{1}\right) \leq \ell\left(\gamma_{0}\right)$, $\sigma_{1} \subset \gamma_{1}$, and, in particular, the above claim holds for $\gamma_{1}$.

As above, we define

$t_{2}:=\sup \left\{t \in[0,1]: l\left(\gamma_{1}(t), x\right)=R_{2}\right\} \quad$ and $\quad s_{2}:=\inf \left\{t \in[0,1]: l\left(\gamma_{1}(t), y\right)=R_{2}\right\}$.

Then $\sigma_{2}:=\left.\gamma_{1}\right|_{\left[t_{2}, s_{2}\right]}$ is a subpath of $\gamma_{1}$ with endpoints $a_{2}:=\gamma_{1}\left(t_{2}\right), b_{2}:=\gamma_{1}\left(s_{2}\right), \sigma_{2}$ lies outside $B\left(x ; R_{2}\right) \cup B\left(y ; R_{2}\right)$, and $\sigma_{1} \subset \sigma_{2}$. We claim that if $\gamma \in \Gamma(x, y)$ satisfies

$$
\sigma_{2} \subset \gamma, \quad \ell(\gamma) \leq b L, \quad \text { and } \quad \ell\left(\gamma\left[x, a_{1}\right]\right) \vee \ell\left(\gamma\left[y, b_{1}\right]\right) \leq b R_{1},
$$


then

$$
\forall z \in \sigma_{2}, \forall w \in\{x, y\}: \quad \ell(\gamma[w, z]) \leq c l(w, z) .
$$

For suppose $\gamma$ is such a path and $z, w$ are such points. If $z$ lies on $\sigma_{1}$, the asserted inequality follows from the claim two paragraphs above. Assume $z$ lies on, say, $\sigma_{2}\left[a_{2}, a_{1}\right]$ (i.e., on $\left.\left.\gamma_{1}\right|_{\left[t_{2}, t_{1}\right]}\right)$. Then $l(x, z) \geq R_{2}$ and so

$$
\ell(\gamma[x, z]) \leq \ell\left(\gamma\left[x, a_{1}\right]\right) \leq b R_{1}=b^{2} R_{2} \leq c l(x, z) .
$$

Also, $l(y, z) \geq R_{1}$, so

$$
\ell(\gamma[y, z]) \leq \ell(\gamma) \leq b L=b^{2} R_{1} \leq c l(y, z) .
$$

If $z$ lies on $\sigma_{2}\left[b_{1}, b_{2}\right]$, we use a similar argument. In particular, the above holds for $\gamma_{1}$.

We modify $\gamma_{1}$ to obtain a path $[0,1] \stackrel{\gamma_{2}}{\rightarrow} X$ in $\Gamma(x, y)$ as follows: $\gamma_{2}$ is the concatenation of paths $\alpha_{2}, \sigma_{2}, \beta_{2}$ where $\alpha_{2}:\left[0, t_{2}\right] \rightarrow X, \beta_{2}:\left[s_{2}, 1\right] \rightarrow X$ are paths in $\Gamma\left(x, a_{2}\right), \Gamma\left(y, b_{2}\right)$ (respectively) which are chosen to have lengths at most $b R_{2}$, and we use appropriate subpaths of $\gamma_{1}$ if these are short enough. Thus $\ell\left(\gamma_{2}\right) \leq \ell\left(\gamma_{1}\right)$, $\sigma_{2} \subset \gamma_{2}$, and in particular the above claim holds for $\gamma_{2}$.

Continuing this process, we construct paths $\gamma_{1}, \ldots, \gamma_{n}$ satisfying

$$
\forall 1 \leq i \leq n: \quad \ell\left(\gamma\left[x, a_{i}\right]\right) \vee \ell\left(\gamma\left[y, b_{i}\right]\right) \leq b R_{i}
$$

with $\gamma_{n}$ being the concatenation of paths $\alpha_{n}, \sigma_{n}, \beta_{n}$ where $\alpha_{n}:\left[0, t_{n}\right] \rightarrow X, \beta_{n}$ : $\left[s_{n}, 1\right] \rightarrow X$ are paths in $\Gamma\left(x, a_{n}\right), \Gamma\left(y, b_{n}\right)$ (respectively) which are chosen to have lengths at most $b R_{n}$, and we use appropriate subpaths of $\gamma_{n-1}$ if these are short enough. Here $a_{n}:=\gamma_{n-1}\left(t_{n}\right), b_{n}:=\gamma_{n-1}\left(s_{n}\right)$ are the last, first (respectively) points of $\gamma_{n-1}$ in the balls $\bar{B}\left(x ; R_{n}\right), \bar{B}\left(y ; R_{n}\right)$ (respectively) and $\sigma_{n}:=\left.\gamma_{n-1}\right|_{\left[t_{n}, s_{n}\right]} \supset \sigma_{n-1}$. Moreover, we find that $\sigma_{n}$ enjoys the property that for any $\gamma \in \Gamma(x, y)$ with

$$
\sigma_{n} \subset \gamma, \ell(\gamma) \leq b L, \quad \text { and } \quad \forall 1 \leq i \leq n-1: \ell\left(\gamma\left[x, a_{i}\right]\right) \vee \ell\left(\gamma\left[y, b_{i}\right]\right) \leq b R_{i},
$$

we have

$$
\forall z \in \sigma_{n}, \forall w \in\{x, y\}: \quad \ell(\gamma[w, z]) \leq \operatorname{cl}(w, z) .
$$

In particular, the above hypotheses - hence the conclusion too-hold for the path $\gamma=\gamma_{n}$.

Now we define

$$
\begin{aligned}
t_{n+1} & :=\sup \left\{t \in[0,1]: l\left(\gamma_{n}(t), x\right)=R_{n+1}\right\}, \\
s_{n+1} & :=\inf \left\{t \in[0,1]: l\left(\gamma_{n}(t), y\right)=R_{n+1}\right\},
\end{aligned}
$$

$\sigma_{n+1}:=\left.\gamma_{n}\right|_{\left[t_{n+1}, s_{n+1}\right]}$ and $a_{n+1}:=\gamma_{n}\left(t_{n+1}\right), b_{n+1}:=\gamma_{n}\left(s_{n+1}\right)$; so $\sigma_{n+1}$ lies outside $B\left(x ; R_{n+1}\right) \cup B\left(y ; R_{n+1}\right)$, and $\sigma_{n} \subset \sigma_{n+1}$. We claim that for any $\gamma \in \Gamma(x, y)$ satisfying

$$
\sigma_{n+1} \subset \gamma, \ell(\gamma) \leq b L, \quad \text { and } \quad \forall 1 \leq i \leq n: \ell\left(\gamma\left[x, a_{i}\right]\right) \vee \ell\left(\gamma\left[y, b_{i}\right]\right) \leq b R_{i},
$$

we have

$$
\forall z \in \sigma_{n+1}, \forall w \in\{x, y\}: \quad \ell(\gamma[w, z]) \leq \operatorname{cl}(w, z)
$$


(E.g., this holds for $\gamma=\gamma_{n}$.) To check this claim, let $\gamma$ be such a path. It suffices to consider points $z$ on $\sigma_{n+1} \backslash \sigma_{n}$. Suppose $z$ lies on $\sigma_{n+1}\left[a_{n+1}, a_{n}\right]$ (or more precisely, on $\left.\left.\gamma_{n}\right|_{\left[t_{n+1}, t_{n}\right]}\right)$. Then $l(x, z) \geq R_{n+1}$, so

$$
\ell(\gamma[x, z]) \leq \ell\left(\gamma\left[x, a_{n}\right]\right) \leq b R_{n}=b^{2} R_{n+1} \leq c l(x, z) .
$$

Also, $l(y, z) \geq R_{1}$, so

$$
\ell(\gamma[y, z]) \leq \ell(\gamma) \leq b L=b^{2} R_{1} \leq c l(y, z) .
$$

If $z$ lies on $\sigma_{n+1}\left[b_{n}, b_{n+1}\right]$ we use a similar argument.

Next modify $\gamma_{n}$ to obtain a path $[0,1] \stackrel{\gamma_{n+1}}{\rightarrow} X$ in $\Gamma(x, y)$ by taking $\gamma_{n+1}$ to be the concatenation of paths $\alpha_{n+1}, \sigma_{n+1}, \beta_{n+1}$ where $\alpha_{n+1}:\left[0, t_{n+1}\right] \rightarrow X, \beta_{n+1}$ : $\left[s_{n+1}, 1\right] \rightarrow X$ are paths in $\Gamma\left(x, a_{n+1}\right), \Gamma\left(y, b_{n+1}\right)$ (respectively) which are chosen to have lengths at most $b R_{n+1}$, and we use appropriate subpaths of $\gamma_{n}$ if these are short enough. Then $\ell\left(\gamma_{n+1}\right) \leq \ell\left(\gamma_{n}\right), \sigma_{n+1} \subset \gamma_{n+1}$, and $\ell\left(\gamma_{n+1}\left[x, a_{n+1}\right]\right) \vee$ $\ell\left(\gamma_{n+1}\left[y, b_{n+1}\right]\right) \leq b R_{n+1}$. Thus the above process can be continued ad infinitum.

Finally, set $t=\lim t_{n}, s=\lim s_{n}$ and define $[t, s] \stackrel{\gamma}{\rightarrow} X$ by $\gamma(t)=x, \gamma(s)=y$ and for $t \in\left[t_{n}, s_{n}\right], \gamma(t)=\sigma_{n}(t)$.

2.B. Quasiconvexity. A rectifiable path $\gamma$, with endpoints $x, y$, is $c$-quasiconvex, $c \geq 1$, if its length is at most $c$ times the distance between its endpoints; i.e., if $\gamma$ satisfies

$$
\ell(\gamma) \leq c|x-y|
$$

A metric space is c-quasiconvex if each pair of points can be joined by a $c$-quasiconvex path. A 1-quasiconvex metric space is usually called geodesic, and a space is a length space if and only if it is $c$-quasiconvex for all $c>1$. By cutting out any loops, we can always replace a $c$-quasiconvex path with a $c$-quasiconvex arc having the same endpoints; see [Väi94].

Quasiconvex spaces are precisely the spaces which are bilipschitz equivalent to length spaces. Indeed, the identity map id $:(X, l) \rightarrow(X, d)$ is always Lipschitz continuous because $|x-y| \leq l(x, y)$ for all $x, y$. Evidently, $X$ is quasiconvex if and only if this identity map is bilipschitz. In particular, if $X$ is $c$-quasiconvex, this map is $c$-bilipschitz.

Examples of quasiconvex spaces include quasiextremal distance Euclidean domains and more generally upper regular Loewner spaces and doubling metric measure spaces which support a $(1, p)$-Poincaré inequality. This list includes Carnot groups and certain Riemannian manifolds with non-negative Ricci curvature; see [GM85, 2.7] and [HK98, 3.13, §6].

It is important to know when the identity map $(X, l) \stackrel{\mathrm{id}}{\rightarrow}(X, d)$ is a homeomorphism; see the end of $\S 2$.C. We show below that this is true precisely when $(X, d)$ enjoys a 'weak local quasiconvexity' property. For later applications, we declare a minimally nice space $(X, d)$ to be locally $(c, \lambda)$-quasiconvex provided $c \geq 1,0<\lambda \leq 1 / 2$, and for all $x \in X$, each pair of points in $\lambda B(x)$ can be joined with a $c$-quasiconvex path. Thus in such a space, the map id $:(X, l) \rightarrow(X, d)$ is uniformly locally $c$-bilipschitz.

2.2. Proposition. For a rectifiably connected space, the identity map $(X, l) \stackrel{\text { id }}{\rightarrow}$ $(X, d)$ is a homeomorphism if and only if each point $x \in X$ has an open neighborhood 
$U$ and an associated homeomorphism $\varphi:[0, \infty) \rightarrow[0, \infty)$ such that

$$
\forall y \in U: \quad \exists \gamma \in \Gamma(x, y) \quad \text { with } \quad \ell(\gamma) \leq \varphi(|x-y|) .
$$

Proof. Since the identity map $(X, l) \stackrel{\text { id }}{\rightarrow}(X, d)$ is always Lipschitz, it suffices to check that the stated condition is equivalent to continuity of its inverse. For this we may consider a fixed point $x \in X$. The sufficiency is transparent; we verify the necessity. So, assume id $:(X, d) \rightarrow(X, l)$ is continuous at $x$.

For each $\varepsilon>0, I(\varepsilon):=\{\delta>0:|z-x| \leq \delta \Longrightarrow l(z, x) \leq \varepsilon\} \neq \emptyset$. In fact, $I(\varepsilon)$ is an interval. Put $\sigma(0)=0$ and for $\varepsilon>0$ define $\sigma(\varepsilon):=\sup I(\varepsilon) \cap[0, \varepsilon]$. We note that $\sigma:[0, \infty) \rightarrow[0, \infty)$ is increasing and right-continuous. From this we find that

$$
\psi(s):=\sup \sigma^{-1}[0, s]
$$

is also increasing and right-continuous with the property that $\sigma(\psi(s)) \geq s$ for all $s \geq 0$.

Put $t=|x-y|$ and $\varepsilon=\psi(2 t)$. Then $|x-y|=t<2 t \leq \sigma(\psi(2 t))=\sigma(\varepsilon)$, so by definition of $\sigma$ we deduce that $l(x, y) \leq \varepsilon=\psi(2 t)=\psi(2|x-y|)$. Thus it remains to replace $\psi$ with a homeomorphism $\varphi:[0, \infty) \rightarrow[0, \infty)$ satisfying $\varphi(t)>\psi(2 t)$.

To this end, put $t_{n}=2^{n}$ (for $n=0,1, \ldots$ ) and select $s_{1}=1>s_{2}>\cdots>$ $s_{n} \searrow 0$ so that $\psi\left(s_{n}\right)$ are all distinct. (For example, put $s_{n+1}:=\sup \left[0,1 / 2^{n}\right] \cap$ $\psi^{-1}\left[0, \psi\left(s_{n}\right) / 2\right]$.) Now let $\Psi$ be the piecewise linear function determined by setting

$$
\forall n \geq 2, \quad \Psi\left(s_{n}\right):=\psi\left(s_{n-1}\right) \quad \text { and } \quad \forall n \geq 0, \quad \Psi\left(t_{n}\right):=\psi\left(t_{n+1}\right)+2^{n} .
$$

Note that $\Psi$ is a homeomorphism which strictly dominates $\psi$. Then $\varphi(t)=\Psi(2 t)$ 'works'.

Minor modifications to the above argument reveal that $(X, d) \stackrel{\text { id }}{\rightarrow}(X, l)$ is locally uniformly continuous (e.g., this holds for continuous maps on locally compact spaces) if and only if $X$ is 'uniformly weakly locally quasiconvex' meaning that each point $z \in X$ has an open neighborhood $U$ and an associated homeomorphism $\varphi:[0, \infty) \rightarrow[0, \infty)$ such that

$$
\forall x, y \in U: \quad \exists \gamma \in \Gamma(x, y) \quad \text { with } \quad \ell(\gamma) \leq \varphi(|x-y|) .
$$

As a simple example, let $X$ be the set in $\mathbf{R}^{2}$ defined as $[(0,0),(1,0)]$ together with the union over all positive integers $n$ of the line segments joining the origin to the points $(1,1 / n)$ and take $d$ to be Euclidean distance. Then $X$ is not 'weakly locally quasiconvex' at any point of $((0,0),(1,0)]$, it is 'weakly locally quasiconvex' at the origin, but not uniformly so.

2.C. Quasihyperbolic distance. The quasihyperbolic distance between two points $x, y$ in a minimally nice space $(X, d)$ is defined by

$$
k(x, y)=k_{X}(x, y):=\inf _{\gamma \in \Gamma(x, y)} \ell_{k}(\gamma) \quad \text { where } \quad \ell_{k}(\gamma):=\inf _{\gamma \in \Gamma(x, y)} \int_{\gamma} \frac{|d z|}{d(z)} ;
$$

here $|d z|$ denotes $d$-arclength. Note that $(X, k)$ is a length space. Quasihyperbolic geodesics always exist in locally compact spaces. However, in general, quasihyperbolic geodesics may not exist; see [Väi99, 3.5] for an example due to P. Alestalo.

We do not assume local compactness. The role of quasihyperbolic geodesics is subsumed by quasihyperbolically short arcs: a path $\gamma \in \Gamma(x, y)$ is called $h$-short provided $h \geq 0$ and $\ell_{k}(\gamma) \leq k(x, y)+h$. We note that every subpath of an $h$-short path is again $h$-short. 
We remind the reader of the following basic estimates for quasihyperbolic distance, first established by Gehring and Palka [GP76, 2.1]:

$$
k(x, y) \geq \log \left(1+\frac{l(x, y)}{d(x) \wedge d(y)}\right) \geq j(x, y):=\log \left(1+\frac{|x-y|}{d(x) \wedge d(y)}\right) \geq\left|\log \frac{d(x)}{d(y)}\right| .
$$

See also [BHK01, (2.3), (2.4)]. The first inequality above is a special case of the more general (and easily proved) inequality

$$
\ell_{k}(\gamma) \geq \log \left(1+\ell(\gamma) / \min _{z \in \gamma} d(z)\right)
$$

which holds for any rectifiable path $\gamma$ in $X$. From the above estimates, we readily get the following, where $\gamma$ is a rectifiable path in $X, u, v \in \gamma$, and $K=\ell_{k}(\gamma)$ :

$$
\begin{gathered}
v \in\left(e^{K}-1\right) \bar{B}(u), \quad 1-e^{-K} \leq \frac{\ell(\gamma)}{d(u)} \leq e^{K}-1, \\
e^{-K} \leq \frac{d(v)}{d(u)} \leq e^{K}, \quad e^{-K} \leq \frac{\ell(\gamma) / d(u)}{\ell_{k}(\gamma)} \leq e^{K} .
\end{gathered}
$$

It follows from the above that the identity map $(X, k) \stackrel{\mathrm{id}}{\rightarrow}(X, d)$ is continuous. Note that this map is a homeomorphism if and only if id : $(X, l) \rightarrow(X, d)$ is a homeomorphism; see [BHK01, A.4, p. 92] (which says that the $k$ - and $l$-topologies coincide). Proposition 2.2 provides a criterion for this to hold. The importance of this property is its usefulness in demonstrating that $(X, k)$ is complete, provided id $:(X, d) \rightarrow(X, l)$ is continuous. This is explained in [BHK01, 2.8], but perhaps worth mentioning here as well. The inequalities given above reveal that every Cauchy sequence in $(X, k)$ is Cauchy in $(X, d)$ and cannot converge to $\partial X$.

\section{UNIFORM SPACES}

Roughly speaking, a space is uniform when points in it can be joined by paths which are not too long and which move away from the regions boundary. A minimally nice metric space $(X, d)$ is called a uniform space provided there is some constant $c \geq 1$ such that each pair of points can be joined by a $c$-uniform path. A rectifiable path $\gamma$ joining $x, y$ in $X$ is a $c$-uniform path provided

$$
\ell(\gamma) \leq c|x-y|
$$

and

$$
\forall z \in \gamma: \quad \ell(\gamma[x, z]) \wedge \ell(\gamma[y, z]) \leq c d(z) .
$$

We call $\gamma$ a double c-cone path if it satisfies the second inequality above (the phrases cigar path and corkscrew are also used). In [Väi88], Väisälä provides a description of various possible double cone conditions (which he calls length cigars, diameter cigars, distance cigars, and Möbius cigars). The work [Mar80] of Martio should also be mentioned.

When our uniform space is an Euclidean domain with Euclidean distance, we call it a uniform domain. Uniform domains in Euclidean space were first studied by John [Joh61] and Martio and Sarvas [MS79] who proved injectivity and approximation results for them. They are well recognized as being the 'nice' domains for quasiconformal function theory as well as many other areas of geometric analysis (e.g., potential theory); see [Geh87] and [Väi88]. Every plane uniform domain is a quasicircle domain (each of its boundary components is either a point or a quasicircle), and a finitely connected plane domain is uniform if and only if it is a quasicircle 
domain. However, the plane punctured at the integers is not uniform. Such nice topological information is not true for uniform domains in higher dimensions. For example, a ball with a radius removed is uniform; this is not true in dimension $n=2$.

For domains in $\mathbf{R}^{n}$ we can consider uniformity both with respect to Euclidean distance and also with respect to the induced length metric. The latter class of domains are usually called inner uniform; cf. [Väi98]. For example, a slit disk in the plane is not uniform (with respect to Euclidean distance) but it is an inner uniform domain. An infinite strip, or the inside of an infinite cylinder in space, is not uniform nor inner uniform. The region between two parallel planes is not uniform nor inner uniform. Every quasiball is uniform.

Uniform subdomains of Heisenberg groups and the more general Carnot groups have become a focus of study; see [CT95], [CGN00], [Gre01]. Bonk, Heinonen, and Koskela [BHK01] introduced the notion of uniformity in the locally compact metric space setting and showed that there is a two way correspondence between these spaces and the so-called Gromov hyperbolic proper geodesic spaces. Note that here we do not assume our uniform spaces are locally compact.

Now we present a metric space version of the Gehring-Osgood-Väisälä characterization of uniform domains. In the Euclidean setting, Gehring and Osgood [GO79] demonstrated that these are precisely the domains having quasihyperbolic distance bilipschitz equivalent to the $j$ distance (defined in the basic distance estimates given in $\S 2 . \mathrm{C}$ ). It turns out that the following seemingly weaker condition also characterizes uniformity. For uniform subdomains of Banach spaces, this result is due to Väisälä [Väi91, 6.16, 6.17]. Bonk, Heinonen, and Koskela [BHK01, 2.13] established the necessary condition, $k \leq 4 c^{2} j$; while stated for locally compact $c$-uniform spaces, their proof does not use local compactness.

We denote the so-called relative distance between $x, y$ by $r(x, y):=\frac{|x-y|}{d(x) \wedge d(y)}$.

3.1. Theorem. A minimally nice locally $(a, \lambda)$-quasiconvex space $(X, d)$ is uniform if and only if there is a homeomorphism $\vartheta:[0, \infty) \rightarrow[0, \infty)$ satisfying $\lim \sup _{t \rightarrow \infty} \vartheta(t) / t<1$ and such that for all points $x, y \in X, k(x, y) \leq \vartheta(r(x, y))$. The uniformity constant depends only on $\vartheta, a$, and conversely in a c-uniform space, one can always take $\vartheta(t)=4 c^{2} \log (1+t)$.

Proof. That uniform spaces satisfy such a condition is the content of [BHK01, 2.13]. Therefore, it suffices to prove the converse. Assume $k \leq \vartheta \circ r$ where $\vartheta$ has the asserted properties. Put $\eta=\lambda / a$, fix $1<\Lambda<\left(\lim \sup _{t \rightarrow \infty} \vartheta(t) / t\right)^{-1}$ and choose $T=T(\vartheta)>1$ so that

$$
1+T^{-1}<\Lambda^{1 / 2} \quad \text { and } \quad \forall t \geq T: \quad \vartheta(\Lambda t) \leq t .
$$

Now let $x, y \in X$. Suppose $d(x) \leq d(y)$; so $r(x, y)=|x-y| / d(x)$. We consider three cases:

$$
|x-y|<\eta d(x), \quad \text { or } \quad|x-y| \geq \eta d(x) \quad \text { and } \quad k(x, y) \leq T, \quad \text { or } \quad k(x, y) \geq T .
$$

Suppose $|x-y|<\eta d(x)$. Let $\gamma \in \Gamma(x, y)$ be an $a$-quasiconvex path. Then $\gamma \subset \lambda B(x)$, so for all $z \in \gamma$,

$$
d(z) \geq(1-\lambda) d(x) \geq(1-\lambda)|x-y| / \eta \geq(1-\lambda) \ell(\gamma) / \lambda ;
$$

since $\lambda \in(0,1 / 2], \lambda /(1-\lambda) \leq 1$ and we see that $\gamma$ is an $a$-uniform path. 
Suppose $|x-y| \geq \eta d(x)$ and $k(x, y) \leq T$. Let $N$ be the smallest integer larger than $2 T / \log (1+\eta)$. Select $\gamma \in \Gamma(x, y)$ with $\ell_{k}(\gamma) \leq 2 k(x, y)$. We show that $\gamma$ is a $c$-quasiconvex double $b$-cone path with $c=\sum_{i=1}^{N}(1+\eta)^{i}$ and $b=\eta(1+\eta)^{N} c$. Thus, e.g., $\gamma$ is a $4 e^{4 T}$-uniform path. We start by dividing $\gamma$ into $N$ subpaths $\gamma_{i}:=$ $\gamma\left[x_{i-1}, x_{i}\right](1 \leq i \leq N)$ of equal quasihyperbolic length, so $\gamma$ is the concatenation of $\gamma_{1}, \ldots, \gamma_{N}$ and

$$
\forall 1 \leq i \leq N: \quad k\left(x_{i}, x_{i-1}\right) \leq \ell_{k}\left(\gamma_{i}\right)=\frac{\ell_{k}(\gamma)}{N} \leq \frac{2 T}{N}<\log (1+\eta) ;
$$

here $x=x_{0}, x_{1}, \ldots, x_{N}=y$ are successive points along $\gamma$.

From the above, and the basic distance estimates (cf. §2.C), we see that for each $1 \leq i \leq N$,

$$
\log (1+\eta)>\ell_{k}\left(\gamma_{i}\right) \geq \log \left(1+\ell\left(\gamma_{i}\right) / d\left(x_{i}\right)\right) \geq\left|\log d\left(x_{i}\right) / d\left(x_{i-1}\right)\right| .
$$

Thus, for each $1 \leq i \leq N$,

$$
\ell\left(\gamma_{i}\right) \leq \eta d\left(x_{i}\right) \quad \text { and } \quad(1+\eta)^{-1} \leq \frac{d\left(x_{i}\right)}{d\left(x_{i-1}\right)} \leq(1+\eta) .
$$

Recalling that $|x-y| \geq \eta d(x)$ we now deduce that

$$
\ell(\gamma)=\sum_{i=1}^{N} \ell\left(\gamma_{i}\right) \leq \eta \sum_{i=1}^{N} d\left(x_{i}\right) \leq \eta \sum_{i=1}^{N}(1+\eta)^{i} d(x) \leq c|x-y|
$$

as desired. To check the asserted double cone condition, we note that for any $z \in \gamma_{i}$,

$$
d(z) \geq d\left(x_{i-1}\right) /(1+\eta) \geq \cdots \geq d(x) /(1+\eta)^{i} \geq \ell(\gamma) / b .
$$

It remains to examine the case $k(x, y) \geq T$. Note that by our choice of $T$ :

$$
\vartheta(\Lambda T) \leq T \leq k(x, y) \leq \vartheta(r(x, y)) \Longrightarrow r(x, y) \geq \Lambda T, \text { so }|x-y| \geq \Lambda T d(x) .
$$

Let $\gamma \in \Gamma(x, y)$ be a 1-short path. We demonstrate that $\gamma$ is a $c$-quasiconvex double $b$-cone path where $c=3 \exp (\vartheta(4 b)+1), b=C^{2}\left(2 \Lambda^{1 / 4}-1\right) /\left(\Lambda^{1 / 4}-1\right)$ and $C=\exp (T+1)$. Our argument is based on the following fact. Here and below, $\kappa=(1 / 4) \log \Lambda$.

Claim. Let $\sigma \in \Gamma(z, w)$ be a 1-short path. Suppose $k(z, w) \geq T$ and $d(z) \leq d(w)$. Then there exists a point $v \in \sigma$ such that

$$
d(v) \geq \Lambda^{1 / 4} d(z) \quad \text { and } \quad \kappa \leq k(z, v) \leq T .
$$

To prove this claim, we first show that there exist a point $v \in \sigma$ with $d(v) \geq$ $\Lambda^{1 / 2} d(z)$. (We then apply this repeatedly to subarcs of $\sigma$.) To see this, note (again) that

$$
\vartheta(\Lambda T) \leq T \leq k(z, w) \leq \vartheta(r(z, w)) \Longrightarrow r=r(z, w) \geq \Lambda T, \text { so } r / \Lambda \geq T
$$

and therefore $k(z, w) \leq \vartheta(r) \leq r / \Lambda=|z-w| /[\Lambda d(z)] \leq \ell(\sigma) /[\Lambda d(z)]$. If every $u \in \sigma$ had $d(u)<\Lambda^{1 / 2} d(z)$, then, by our choice of $T$, we would obtain the contradiction

$$
\frac{\ell(\sigma)}{\Lambda^{1 / 2} d(z)} \leq \ell_{k}(\sigma) \leq k(z, w)+1 \leq\left(1+T^{-1}\right) k(z, w)<\Lambda^{1 / 2} \frac{\ell(\sigma)}{\Lambda d(z)} .
$$

We establish the claim for the point $v:=v_{M}$, where a positive integer $M$ and a finite sequence $\left(v_{j}\right)_{j=1}^{M}$ of points on $\sigma$ are defined by a stopping time argument. Applying the initial sub-claim to $\sigma$, we obtain a point $v_{1} \in \sigma$ such that $d\left(v_{1}\right) \geq$ $\Lambda^{1 / 2} d(z)$. Having defined $v_{j}$ for a given integer $j \geq 1$, we stop and declare $M:=j$ 
if $k\left(v_{j}, z\right) \leq T$. Otherwise, the iterative step depends on whether $j$ is odd or even. For odd $j$, we always have $d\left(v_{j}\right) \geq \Lambda^{1 / 2} d(z)$, so the Intermediate Value Theorem ensures that we can find $v_{j+1} \in \sigma\left[z, v_{j}\right]$ such that $d\left(v_{j+1}\right)=\Lambda^{1 / 4} d(z)$. On the other hand, if $j$ is even, then we always have $d\left(v_{j}\right) \geq d(z)$, so we can use the initial sub-claim to find $v_{j+1} \in \sigma\left[z, v_{j}\right]$ with $d\left(v_{j+1}\right) \geq \Lambda^{1 / 2} d(z)$. In either case, using the basic distance estimates, we find that $k\left(v_{j}, v_{j+1}\right) \geq \kappa$, so the process must eventually end, and $v=v_{M}$ satisfies both $d(v) \geq \Lambda^{1 / 4} d(z)$ and $k(z, v) \leq T$. Finally, $k(z, v) \geq|\log d(z) / d(v)| \geq \kappa$.

Now we return to the proof of the theorem. We produce integers $m, n \geq 0$ and successive distinct points $x=x_{0}, x_{1}, \ldots, x_{m}, y_{n}, \ldots, y_{1}, y_{0}=y$ along $\gamma$ with the properties:

$$
\begin{gathered}
\gamma=\bigcup_{i=1}^{m} \alpha_{i} \cup \sigma \cup \bigcup_{j=1}^{n} \beta_{j} ; \\
\alpha_{i}:=\gamma\left[x_{i-1}, x_{i}\right], \beta_{j}:=\gamma\left[y_{j}, y_{j-1}\right], \sigma:=\gamma\left[x_{m}, y_{n}\right] \\
\ell_{k}\left(\alpha_{i}\right) \leq T+1, \ell_{k}\left(\beta_{j}\right) \leq T+1, \ell_{k}(\sigma) \leq T+1 ; \\
d\left(x_{i}\right) \geq \Lambda^{1 / 4} d\left(x_{i-1}\right) \quad \text { and } \quad d\left(y_{j}\right) \geq \Lambda^{1 / 4} d\left(y_{j-1}\right) ;
\end{gathered}
$$

the above conditions being valid for all $1 \leq i \leq m$ and $1 \leq j \leq n$ (when $m>0$ or $n>0)$.

To find these points, we use another stopping time process involving a positive integer $N$ and a finite nested sequence $\left(\gamma_{j}\right)_{j=0}^{N}$ of paths $\gamma_{j}:=\gamma\left[w_{j}, z_{j}\right] \subset \gamma_{j-1}$ for $1 \leq j \leq N$. To begin, we put $w_{0}=x, z_{0}=y$. Given $\gamma_{j}$, we declare $N:=j$ and stop if $k\left(w_{j}, z_{j}\right) \leq T$. When $k\left(w_{j}, z_{j}\right)>T$, we continue: If $d\left(w_{j}\right) \leq d\left(z_{j}\right)$, we apply the Claim to $\gamma_{j}$ to find a point $w_{j+1}$ with $d\left(w_{j+1}\right) \geq \Lambda^{1 / 4} d\left(w_{j}\right)$ and $\kappa \leq k\left(w_{j}, w_{j+1}\right) \leq$ $T$; in this case, we set $z_{j+1}=z_{j}$. If instead $d\left(z_{j}\right) \leq d\left(w_{j}\right)$, we apply the Claim to get a point $z_{j+1}$ with $d\left(z_{j+1}\right) \geq \Lambda^{1 / 4} d\left(z_{j}\right)$ and $\kappa \leq k\left(z_{j}, z_{j+1}\right) \leq T$; now we set $w_{j+1}=w_{j}$. Since $\ell_{k}\left(\gamma_{j+1}\right) \leq \ell_{k}\left(\gamma_{j}\right)-\kappa$, the process eventually ends.

The desired points $x_{i}, y_{j}$ are now obtained by appropriately relabeling the points $w_{k}, z_{k}$. Note that we have the following inequalities which are valid for all $1 \leq i$ $\leq m$ :

$$
\begin{gathered}
\forall 1 \leq k \leq i, \quad d\left(x_{k}\right) \leq \Lambda^{(k-i) / 4} d\left(x_{i}\right) ; \\
\forall z \in \alpha_{i}, \quad C^{-1} \leq d(z) / d\left(x_{i}\right) \leq C ; \\
\ell\left(\alpha_{i}\right) \leq C d\left(x_{i}\right) ;
\end{gathered}
$$

where $C=e^{T+1}$. There are similar inequalities for the points $y_{j}$ and the paths $\beta_{j}$, and for $\sigma$.

To show that $\gamma$ satisfies a double cone condition, suppose $z \in \alpha_{k}$ for some $1 \leq k \leq m$. Then $d(z) \geq d\left(x_{k}\right) / C$ and thus

$$
\ell(\gamma[x, z]) \leq \sum_{i=1}^{k} \ell\left(\alpha_{i}\right) \leq C \sum_{i=1}^{k} \Lambda^{(i-k) / 4} d\left(x_{k}\right) \leq \frac{C \Lambda^{1 / 4}}{\Lambda^{1 / 4}-1} d\left(x_{k}\right) \leq b d(z) .
$$

A similar argument applies to points on any $\beta_{k}$, so it remains to consider a point $z \in \sigma$. From what was just established we have

$$
\ell\left(\gamma\left[x, x_{m}\right]\right) \leq \frac{C \Lambda^{1 / 4}}{\Lambda^{1 / 4}-1} d\left(x_{m}\right) \quad \text { and } \quad \ell\left(\gamma\left[y, y_{n}\right]\right) \leq \frac{C \Lambda^{1 / 4}}{\Lambda^{1 / 4}-1} d\left(y_{n}\right) .
$$


Also, $\ell(\sigma) \leq C\left[d\left(x_{m}\right) \wedge d\left(y_{n}\right)\right]$. Since $d(z) \geq\left[d\left(x_{m}\right) \vee d\left(y_{n}\right)\right] / C$, we conclude that in all cases $\gamma$ satisfies the double $b$-cone condition.

Finally, we prove the quasiconvexity condition. First, note that $|x-y| \geq$ $\frac{1}{2}[d(x) \vee d(y)]$; for if this were not the case, then we would have $k(x, y) \leq \vartheta(1)<T$. Now pick $x^{\prime}, y^{\prime} \in \gamma$ so that $\ell\left(\gamma\left[x, x^{\prime}\right]\right)=|x-y| / 2=\ell\left(\gamma\left[y, y^{\prime}\right]\right)$. Then

$$
d\left(x^{\prime}\right) \leq d(x)+\left|x-x^{\prime}\right| \leq(5 / 2)|x-y| \text { and likewise } \quad d\left(y^{\prime}\right) \leq(5 / 2)|x-y| .
$$

Also, the double cone condition ensures that

$$
d\left(x^{\prime}\right) \geq \frac{|x-y|}{2 b} \quad \text { and } \quad d\left(y^{\prime}\right) \geq \frac{|x-y|}{2 b},
$$

so $r\left(x^{\prime}, y^{\prime}\right) \leq 2|x-y| /\left[d\left(x^{\prime}\right) \wedge d\left(y^{\prime}\right)\right] \leq 4 b$. Thus $k\left(x^{\prime}, y^{\prime}\right) \leq \vartheta(4 b)$, so $\ell_{k}\left(\gamma\left[x^{\prime}, y^{\prime}\right]\right) \leq$ $k\left(x^{\prime}, y^{\prime}\right)+1 \leq \vartheta(4 b)+1$. Therefore

$\ell\left(\gamma\left[x^{\prime}, y^{\prime}\right]\right) \leq[\exp (\vartheta(4 b)+1)-1]\left[d\left(x^{\prime}\right) \wedge d\left(y^{\prime}\right)\right] \leq(5 / 2)[\exp (\vartheta(4 b)+1)-1]|x-y|$.

Finally,

$$
\ell(\gamma)=\ell\left(\gamma\left[x, x^{\prime}\right]\right)+\ell\left(\gamma\left[x^{\prime}, y^{\prime}\right]\right)+\ell\left(\gamma\left[y^{\prime}, y\right]\right) \leq 3 \exp (\vartheta(4 b)+1)|x-y| .
$$

3.2. Remarks. (a) One can easily construct spaces with $k \leq \vartheta \circ r$ which fail to be uniform. That is, the local quasiconvexity hypothesis cannot be dropped.

(b) We see from the above that any estimate $k(x, y) \leq \vartheta(r(x, y))$ for $k(x, y) \geq K$ implies uniformity. Indeed, in the first two cases above we find that points $x, y$ in a locally $(a, \lambda)$-quasiconvex space with $k(x, y) \leq K$ can be joined by a $c$-uniform path where $c=a \vee 4 e^{4 K}$.

(c) An important, and characteristic, property of locally compact uniform spaces is that quasihyperbolic quasigeodesics are uniform arcs. See [GO79] for domains in Euclidean space and [BHK01, 2.10] for general metric spaces, both of which use quasihyperbolic geodesics. Slight alterations to their proofs generalize to the quasigeodesic situation.

As an application of the above theorem, we corroborate that quasihyperbolically short paths are uniform paths. More precisely, we prove the following.

3.3. Corollary. Suppose $(X, d)$ is a c-uniform space. Let $x, y \in X$ and put $h=$ $1 \wedge k(x, y)$. Any h-short path from $x$ to $y$ is a b-uniform path with $b \leq 3 \exp \left(200 c^{6}\right)$.

Proof. Thanks to [BHK01, 2.13], we know that $k \leq \vartheta \circ r$ where $\vartheta(t)=4 c^{2} \log (1+t)$. Put $\Lambda=81 / 16$, so $\Lambda^{1 / 4}=3 / 2$, and let $T=19 c^{4}$. We claim that for all $t \geq T$, $\vartheta(\Lambda t) \leq t$. This follows from an elementary calculus argument. (Note that for $a>0$, the functions $x \mapsto \log (1+a x) / x$ and $x \mapsto \log \left(1+a x^{2}\right) / x$ are decreasing; the first for all $x>0$, the second for $1+a x^{2} \geq 5$.) Indeed, with the change of variable $t=c^{4} s$ our claim becomes the assertion that for $s \geq 19$,

$$
\frac{4}{s} x^{-1} \log \left(1+a x^{2}\right)=\frac{4}{c^{2} s} \log \left(1+c^{4} \Lambda s\right) \leq 1 \quad \text { where } x=c^{2} \text { and } a=s \Lambda .
$$

Evidently, for all $s \geq 19$ and $c \geq 1,1+a x^{2}=1+c^{4} \Lambda s \geq 5$, so it suffices to prove the above inequality for $c=1$ and all $s \geq 19$. For $s \geq 19, s^{-1} \log (1+\Lambda s) \leq$ $19^{-1} \log (1+19 \Lambda)$, so

as asserted.

$$
\frac{4}{s} \log (1+\Lambda s) \leq \frac{4}{19} \log (1+19 \Lambda) \leq 1
$$


Now suppose $\gamma \in \Gamma(x, y)$ is an $h$-short path. As in the proof of Theorem 3.1, we consider three cases. In the first case, where $|x-y| \leq \eta d(x)$ with $\eta=1 / 2 c$ and $d(x) \leq d(y)$, we select a $c$-quasiconvex $\alpha \in \Gamma(x, y)$ to deduce that

$$
k(x, y) \leq \ell_{k}(\alpha) \leq \frac{c|x-y|}{d(x) / 2} \leq 1, \quad \text { so } \quad \ell_{k}(\gamma) \leq 2 .
$$

From the last inequality in $\S 2$.C we obtain

$$
\frac{\ell(\gamma)}{d(x)} \leq e^{k(x, y)} \ell_{k}(\gamma) \leq 4 c e \frac{|x-y|}{d(x)}
$$

so $\gamma$ is 4 ce-quasiconvex; also, $\ell(\gamma) \leq 2 e d(x)$. Next,

$$
\forall z \in \gamma: \quad d(z) \geq e^{-k(x, z)} d(x) \geq e^{-3} \ell(\gamma) / 2,
$$

which says that $\gamma$ is a double $e^{3}$-cone path and thus, e.g., a $4 c e^{2}$-uniform path.

In the second case, where $|x-y| \geq \eta d(x)$ and $k(x, y) \leq T$, our proof of Theorem 3.1 reveals that $\gamma$ is a $b$-uniform path with $b=4 \exp (4 T)=4 \exp \left(72 c^{4}\right)$. In the final case, where $k(x, y) \geq T$, our proof indicates that $\gamma$ is $b$-uniform now with $b=3 \exp \left(\vartheta\left(16 C^{2}\right)+1\right)$ and $C=\exp (T+1)=\exp \left(19 c^{4}+1\right)$. As $\log \left(1+16 C^{2}\right) \leq \log 17+2 \log C \leq(2+\log 17)+2 T$, we obtain

$$
\vartheta\left(16 C^{2}\right)+1 \leq 1+4 c^{2}[(2+\log 17)+2 T] \leq 1+20 c^{2}+152 c^{6} \leq 173 c^{6} .
$$

\section{SLice CONDitions}

We begin with a few geometric conditions, each with an associated parameter. A space $(X, d)$ is c-linearly locally connected, or $c-L L C$, if $c \geq 1$ and the following two conditions hold for all $x \in X$ and all $r>0$ :

$\left(\mathrm{LLC}_{1}\right)$

$$
\text { points in } B(x ; r) \text { can be joined in } B(x ; c r)
$$

and

$\left(\mathrm{LLC}_{2}\right) \quad$ points in $X \backslash \bar{B}(x ; r)$ can be joined in $X \backslash \bar{B}(x ; r / c)$.

Here the phrase 'can be joined' means 'can be joined by a continuum'. We also employ the terminology $L L C$ with respect to paths in which case 'can be joined' means 'can be joined by a rectifiable path'. Note that quasiconvexity implies LLC $_{1}$ with respect to paths (but not conversely). These conditions, first introduced by Gehring to characterize quasidisks, are well known in the literature.

The generic example of a space which does not satisfy the $\mathrm{LLC}_{2}$ condition is the interior of an infinite Euclidean cylinder such as $\mathbf{B}^{n-1} \times \mathbf{R} \subset \mathbf{R}^{n}$. However, for $2 \leq k<n$ the regions $\mathbf{B}^{n-k} \times \mathbf{R}^{k} \subset \mathbf{R}^{n}$ are easily seen to be $1-L L C_{2}$. The complement of a semi-infinite slab (e.g., $\mathbf{R}^{n} \backslash\left\{\left(x_{1}, \ldots, x_{n}\right): x_{1} \geq 0,\left|x_{n}\right| \leq 1\right\}$ ) fails to be $\mathrm{LLC}_{1}$. Uniform domains are LLC, however uniform spaces may fail to be $\mathrm{LLC}_{2}$ (e.g., a metric tree). The so-called quasiextremal distance (aka, QED) Euclidean domains and more generally Ahlfors regular Loewner spaces are quasiconvex and LLC with respect to paths; see [GM85, 2.7] and [HK98, 3.13].

When a quasiconvex or LLC space is an Euclidean domain $D \subsetneq \mathbf{R}^{n}$, we call $D$ a quasiconvex domain or an LLC domain.

We say that a locally complete metric space $(X, d)$ is c-locally externally connected, abbreviated $c$-LEC, provided $c \geq 1$ and the $\mathrm{LLC}_{2}$ with respect to paths property holds for all points $x \in \Omega$ and all $r \in(0, d(x) / c)$. Every Euclidean domain, in $\mathbf{R}^{n}$ with $n \geq 2$, is $c$-LEC for all $c>1$. 
There are various so-called slice conditions each designed to handle their own specific problem. The exposition [Buc03] is a good place to begin reading about this topic. The first author, and his many co-authors, have utilized an assortment of slice conditions to investigate a number of different questions.

A non-empty bounded open set $S \subset X$ is a $C$-slice separating $x, y$ provided $C \geq 2$ and

$$
C^{-1} B(x) \cap S=\emptyset=S \cap C^{-1} B(y)
$$

and

$$
\forall \alpha \in \Gamma(x, y): \quad \ell(\alpha \cap S) \geq C^{-1} \operatorname{diam}(S) .
$$

A set of $C$-slices for $x, y \in X$ is a collection $\mathcal{S}$ of pairwise disjoint $C$-slices separating $x, y$ in $X$. One can show (see $[\mathrm{BS} 03,2.9]$ ) that in Euclidean spaces, and more generally in $C$-LEC spaces, the cardinality of any such set $\mathcal{S}$ of $C$-slices separating $x, y$ is always bounded by card $\mathcal{S} \leq 4 C^{3} k(x, y)$. We are interested in knowing when we can reverse this inequality. Since there may be no $C$-slices separating $x, y$, we consider the quantity

$$
d_{\mathrm{ws}}(x, y)=d_{\mathrm{ws}}(x, y ; C)=d_{\mathrm{ws}}^{X}(x, y ; C):=1+\sup \operatorname{card} \mathcal{S}
$$

where the supremum is taken over all $\mathcal{S}$ which are sets of $C$-slices in $X$ separating $x, y$.

We call $(X, d)$ a weak $C$-slice space provided for all $x, y \in X: k(x, y) \leq$ $C d_{\mathrm{ws}}(x, y ; C)$. Thus in these spaces $d_{\mathrm{ws}}(x, y) \simeq k(x, y)$ when $k(x, y) \geq 1$. The weak slice condition was introduced in [BO99, Section 5]; see also [BS03], [Buc03] and [Buc04]. When the weak slice space is a domain $D \subsetneq \mathbf{R}^{n}$, we call $D$ a weak slice domain.

As a simple example we note that, when $x$ and $y$ are sufficiently far apart (e.g., say, $|x-y| \geq d(x)+d(y) \geq 2 d(x)$ ), the concentric rings $2^{i} \bar{B}(x) \backslash 2^{i-1} B(x)$ (with $\left.0 \leq i \leq m=\left\lfloor\log _{2}(|x-y| / d(x))\right\rfloor-1\right)$ are 4-slices separating $x, y$. This provides an easy lower estimate for $d_{\mathrm{ws}}(x, y)$ which in turn can be used to check that every $a$-uniform space is a weak $C$-slice space with $C=C(a)$. See [BS03, (2.3)] for details.

The following technical lemma is quite useful for obtaining an upper bound for the cardinality of a set of slices; in weak slice spaces it thus provides an upper bound for quasihyperbolic distances. It is the case $\alpha=0$ of [BS03, 2.17].

4.1. Lemma. Let $A$ be a 1-rectifiable subset of a rectifiably connected metric space $(X, d)$. Suppose $\varphi: A \rightarrow[\varepsilon, \infty)$ (with $\varepsilon>0$ ) and $\mathcal{S}$ is a collection of disjoint non-empty bounded subsets of $X$. Suppose also that there exist positive constants $b, c$ such that

(a) $\forall S \in \mathcal{S}: \quad \ell(S \cap A) \geq c \operatorname{diam}(S)$,

(b) $\forall S \in \mathcal{S}, \forall z \in S \cap A: \quad \varphi(z) \leq \operatorname{diam}(S)$,

(c) $\forall t>0: \quad \ell\left(\varphi^{-1}(0, t]\right) \leq b t$.

Then the cardinality of $\mathcal{S}$ is at most card $\mathcal{S} \leq 2(b / c) \log _{2}(4 \ell(A) / c \varepsilon)$.

Now we present our main result.

4.2. Theorem. A minimally nice metric space $(X, d)$ is uniform and LEC if and only if it is quasiconvex, LLC with respect to paths, and satisfies a weak slice condition. These implications are quantitative.

Proof. Uniformity trivially implies quasiconvexity (and so $\mathrm{LLC}_{1}$ with respect to paths). Also, as mentioned above, uniform spaces are weak slice spaces too. We 
show that an $a$-uniform $b$-LEC space is $c-\mathrm{LLC}_{2}$ with respect to paths, where $c=$ $1+2 a b^{2}$. To this end, fix $z \in X, r>0$ and $x, y \in X \backslash \bar{B}(z ; r)$. Let $\gamma$ be an $a$-uniform path from $x$ to $y$. We assume there is some $u \in \gamma \cap \bar{B}(z ; r / c)$, since otherwise there is nothing to prove. Then

$$
\ell(\gamma[x, u]) \wedge \ell(\gamma[y, u])>r-r / c=(1-1 / c) r,
$$

so uniformity implies that $d(u) \geq[(c-1) / a c] r$. Setting $s:=[(c-1) / a b c] r$ we find that

$$
s<d(u) / b, \quad x, y \notin \bar{B}(z ; s) \quad \text { and } \quad B(z ; r / c) \subset B(u ; s / b) .
$$

Utilizing the LEC property we obtain a path $\alpha$ which joins $x, y$ in $X \backslash \bar{B}(u ; s / b)$ and hence in $X \backslash \bar{B}(z ; r / c)$.

Now we verify the converse. Suppose $(X, d)$ is $a$-quasiconvex, $b$-LLC with respect to paths, and $C$-weak slice. The LEC property follows trivially from $\mathrm{LLC}_{2}$ with respect to paths. Thus it suffices to show that $X$ is $c$-uniform with $c=c(a, b, C)$.

Let $x, y \in X$ with, say, $|x-y|>C^{-1}[d(x)+d(y)]$ (cf. Remark 3.2(b)). Appealing to Proposition 2.1, we select a path $\gamma \in \Gamma(x, y)$ satisfying

$$
\ell(\gamma) \leq 2 l(x, y) \text { and } \forall z \in \gamma: \ell(\gamma[x, z]) \leq 4 l(x, z), \ell(\gamma[y, z]) \leq 4 l(y, z) .
$$

Suppose $\mathcal{S}$ is a set of $C$-slices separating $x$ and $y$. We use Lemma 4.1 to estimate the cardinality of $\mathcal{S}$. Define $A:=\gamma \backslash\left[C^{-1} B(x) \cup C^{-1} B(y)\right], \varepsilon:=[d(x) \wedge d(y)] /(a b C)$ and

$$
\text { for } z \in A \text {, let } \varphi(z):=[l(x, z) \wedge l(y, z)] / a b \text {. }
$$

Note that by definition, $\varphi: A \rightarrow[\varepsilon, \infty)$. Also, requirement (a) in Lemma 4.1 follows with $c=1 / C$ directly from the fact that each $S \in \mathcal{S}$ is a $C$-slice separating $x$ and $y$. Next, by our choice of $\gamma$ we see that if $z \in A$ with $\varphi(z) \leq t$, then $\ell(\gamma[x, z]) \leq 4 a b t$ and $\ell(\gamma[y, z]) \leq 4 a b t$. Thus $\varphi^{-1}((0, t])$ is contained in the union of two end subpaths of $\gamma$ and $\ell\left(\varphi^{-1}((0, t])\right) \leq 8 a b t$.

It remains to check requirement (b) in Lemma 4.1. Fix an $S \in \mathcal{S}$ and let $z \in S \cap A$. By quasiconvexity, $|x-z| \wedge|y-z| \geq[l(x, z) \wedge l(y, z)] / a$. Thus for each $r \in(0,[l(x, z) \wedge l(y, z)] / a), x, y \notin \bar{B}(z ; r)$. Fix such an $r$. Employing the $\mathrm{LLC}_{2}$ hypothesis, we find a rectifiable path $\alpha$ joining $x, y$ in $X \backslash \bar{B}(z ; r / b)$. Since $z \in S$, and $S$ intersects $\alpha$, we deduce that $\operatorname{diam}(S) \geq r / b$. Letting $r$ increase to $[l(x, z) \wedge l(y, z)] / a$ we obtain $\operatorname{diam}(S) \geq \varphi(z)$, as required.

An appeal to Lemma 4.1 now provides the cardinality estimate

$$
\operatorname{card} \mathcal{S} \leq 16 a b C \log _{2} \frac{4 a b C^{2} l(x, y)}{d(x) \wedge d(y)}
$$

where we have used the information that $\ell(A) \leq \ell(\gamma) \leq 2 l(x, y)$. Another application of quasiconvexity, along with the weak $C$-slice condition, yields

$$
k(x, y) \leq C d_{\mathrm{ws}}(x, y ; C) \leq C+16 a b C \log _{2}\left(\frac{8 a^{2} b C^{2}|x-y|}{d(x) \wedge d(y)}\right) \leq C+C^{\prime} \log r(x, y) .
$$

Finally, Theorem 3.1 permits us to conclude that this implies uniformity.

Proof of Theorem in Introduction. Euclidean uniform domains are LLC with respect to paths, quasiconvex, and satisfy a weak slice condition. Since Euclidean domains are LEC, Theorem 4.2 asserts that these three conditions together imply uniformity. 
Proof of Corollary in Introduction. This follows from the comments below once we show that such a domain $D$ is QED. Since $G$ is uniform, it is QED; this follows from Jones' extension result for Sobolev spaces as explained in [GM85, 2.18]. That $D$ is QED now follows automatically because $E$ is $W^{1, n}$-removable.

We finish by discussing some Euclidean domains that explore whether or not we can weaken any of the three conditions equivalent to uniformity in the Theorem in the Introduction. First, it is easy to construct domains that are quasiconvex and LLC with respect to paths but are not weak slice. The key point is that a (nonuniform) QED or Loewner Euclidean domain is quasiconvex and LLC with respect to paths (but is not weak slice); see [GM85, 2.7] or [HK98, 3.13]. In particular, a QED or Loewner domain is uniform if and only if it is weak slice. This topic is further elaborated upon in [BH07].

Next, it is also easy to construct domains that are weak slice and quasiconvex but not LLC. Indeed since all simply connected planar domains are weak slice domains [BS01, 3.1], a simply connected domain with an external cusp suffices as an example. Finally, we do not have an example of a domain that is weak slice and LLC with respect to paths but fails to be quasiconvex. Since $L_{L C}$ is a slightly weakened form of quasiconvexity, it may be that weak slice plus LLC implies quasiconvexity. Note that the slit disk is weak slice and $\mathrm{LLC}_{2}$ with respect to paths.

\section{REFERENCES}

[BHK01] M. Bonk, J. Heinonen, and P. Koskela, Uniformizing Gromov hyperbolic spaces, Astérisque. 270 (2001), 1-99. MR1829896 (2003b:30024)

[Buc03] S. Buckley, Slice conditions and their applications, Future Trends In Geometric Function Theory (Univ. Jyväskylä), vol. 92, Rep. Univ. Jyväskylä Dept. Math. Stat., 2003, RNC Workshop held in Jyväskylä, June 15-18, 2003, pp. 63-76. MR2058111 (2005c:30048)

[Buc04] S.M. Buckley, Quasiconfomal images of Hölder domains, Ann. Acad. Sci. Fenn. Ser. Math. 29 (2004), 21-42. MR2041697 (2004m:30030)

[BH07] S. Buckley and D.A. Herron, Uniform domains and capacity, Israel J. Math 158 (2007), $129-157$.

[BO99] S.M. Buckley and J. O'Shea, Weighted Trudinger-type inequalities, Indiana Univ. Math. J. 48 (1999), 85-114. MR1722194 (2001a:46031)

[BS01] S.M. Buckley and A. Stanoyevitch, Weak slice conditions, product domains, and quasiconformal mappings, Rev. Mat. Iberoam. 17 (2001), 1-37. MR1900897 (2003b:30025)

[BS03] _ Distinguishing properties of weak slice conditions, Conform. Geom. Dyn. 7 (2003), 49-75. MR1992037 (2004f:30014)

[CGN00] L. Capogna, N. Garofalo, and D-M. Nhieu, Examples of uniform and NTA domains in Carnot groups, Proceedings on Analysis and Geometry (Novosibirsk), Izdat. Ross. Akad. Nauk Sib. Otd. Inst. Mat., 2000, (Novosibirsk Akad., 1999), pp. 103-121. MR1847513 (2002k:30037)

[CT95] L. Capogna and P. Tang, Uniform domains and quasiconformal mappings on the Heisenberg group, Manuscripta Math. 86 (1995), no. 3, 267-281. MR1323792 (96f:30019)

[Geh87] F.W. Gehring, Uniform domains and the ubiquitous quasidisk, Jahresber. Deutsch. Math.-Verein 89 (1987), 88-103. MR880189 (88j:30042)

[GM85] F.W. Gehring and O. Martio, Quasiextremal distance domains and extension of quasiconformal mappings, J. Analyse Math. 45 (1985), 181-206. MR833411 (87j:30043)

[GO79] F.W. Gehring and B.G. Osgood, Uniform domains and the quasi-hyperbolic metric, J. Analyse Math. 36 (1979), 50-74. MR581801 (81k:30023)

[GP76] F.W. Gehring and B.P. Palka, Quasiconformally homogeneous domains, J. Analyse Math. 30 (1976), 172-199. MR0437753 (55:10676)

[Gre01] A.V. Greshnov, On uniform and NTA-domains on Carnot groups, Sibirsk. Mat. Zh. 42 (2001), no. 5, 1018-1035. MR1861631 (2002h:53048) 
[HK98] J. Heinonen and P. Koskela, Quasiconformal maps in metric spaces with controlled geometry, Acta. Math. 181 (1998), 1-61. MR1654771 (99j:30025)

[Joh61] F. John, Rotation and strain, Comm. Pure Appl. Math. 14 (1961), 391-413. MR0138225 $(25: 1672)$

[Kos99] P. Koskela, Removable sets for Sobolev spaces, Ark. Mat. 37 (1999), 291-304. MR1714767 (2001g:46077)

[Mar80] O. Martio, Definitions for uniform domains, Ann. Acad. Sci. Fenn. Ser. A I Math. 5 (1980), no. 1, 197-205. MR595191 (82c:30028)

[MS79] O. Martio and J. Sarvas, Injectivity theorems in plane and space, Ann. Acad. Sci. Fenn. Ser. A I Math. 4 (1978/79), no. 2, 383-401. MR565886 (81i:30039)

[Väi88] J. Väisälä, Uniform domains, Tôhoku Math. J. 40 (1988), 101-118. MR927080 (89d:30027)

[Väi91] , Free quasiconformality in Banach spaces II, Ann. Acad. Sci. Fenn. Math. 16 (1991), 255-310. MR1139798 (94c:30028)

[Väi94] Exhaustions of John domains, Ann. Acad. Sci. Fenn. Math. 19 (1994), 47-57. MR1246886 (94i:30024)

[Väi98] R Relatively and inner uniform domains, Conformal Geometry and Dynamics 2 (1998), 56-88. MR1637079 (99e:30014)

[Väi99] The free quasiworld. Freely quasiconformal and related maps in Banach spaces., Quasiconformal Geometry and Dynamics (Lublin, 1996) (Warsaw), vol. 48, Inst. Math., Polish of Academy Sciences, Banach Center Publ., 1999, pp. 55-118.

Department of Mathematics, National University of Ireland, Maynooth, Co. KilDARE, IRELAND

E-mail address: sbuckley@maths.nuim.ie

Department of Mathematics, University of Cincinnati, Cincinnati, Ohio 45221

E-mail address: david.herron@math.uc.edu 